\title{
Active Intuition: The Patterned Spontaneity of Decision-Making
}

\author{
Lawrence Hamilton Williams* \\ Sociology, University of Toronto, Toronto, ON, Canada
}

Sociologists have been split between explaining individual thought and action in terms of factors internal and external to any given individual. In this paper, I argue that while these two explanations may often be complementary, they do not amount to a complete account of how people actually think or act. Using secondary interviews conducted with individuals tasked to think about death and dying, I demonstrate how individuals actively intuit their surroundings using complex mixtures of both the environment that they have internalized as well as the environment (s) that they are currently in. Using the terms cognitive field and active intuition to describe this process of meaning-making, I assert that existing sociological explanations could benefit from taking into consideration both the emergent nature of individuals' perception of the worlds they live in as well as the iterative nature of the interview process.

Keywords: dual-process model, reflexivity, culture, intuition, Emile Durkheim, George Herbert Mead

\section{OPEN ACCESS}

Edited by:

Angelos Mouzakitis, University of Crete, Greece

Reviewed by:

Benjamin W. Kelly,

Nipissing University, Canada Vasiliki Tsakiri,

Hellenic Open University, Greece

*Correspondence:

Lawrence Hamilton Williams lawrence.williams@mail.utoronto.ca

Specialty section:

This article was submitted to Sociological Theory,

a section of the journal

Frontiers in Sociology

Received: 19 July 2018 Accepted: 02 October 2018 Published: 22 October 2018

Citation:

Williams LH (2018) Active Intuition:

The Patterned Spontaneity of

Decision-Making. Front. Sociol. 3:29.

doi: 10.3389/fsoc.2018.00029

\section{INTRODUCTION}

From the increasing use of Bourdieu's (1984) concept of habitus (Bourgois and Schonberg, 2009; Vaisey, 2009) to the more recent use of cognitive schemas (DiMaggio, 1997; Vaisey, 2009; Patterson, 2014), many sociologists have increasingly been trying to explain how the social world influences one's thoughts, actions, and motivations (see Lizardo et al., 2016). Using concepts such as these, many sociologists argue that the social world leaves enduring imprints on individuals' minds which then serve as foundations for these individuals' future actions (Bourgois and Schonberg, 2009; Vaisey, 2009; Lizardo and Strand, 2010).

The idea that focusing on the lasting imprints of experience is a fruitful domain of inquiry for sociologists has been questioned, however, by sociologists who focus on the situated nature of action. For example, Swidler (1986) claims that individuals often act in ways that seem to go against the values that they hold. She claims that they do so either because of the actual opportunities for action that they have (Swidler, 1986) or based on how they happen to assess the situations they confront (Archer, 2007). In this view, people act in contexts, and contexts bear meanings of their own regardless of whatever a person has happened to internalize or believe.

Placing both the focus on internalized cultural residua and the focus on situational constraints together, we are left with two distinct yet somewhat complementary versions of how to go about studying social life. On the one hand, the patterns inherent in the ways in which individuals respond to situations, form lines of action, and conceive of themselves leads to the idea that individuals' most intimate drives and motivations may actually be deeply shaped by tacit, unconscious, deeply internalized cultural residua. According to this view, the culture we share forms some of our deepest and most seemingly personal understandings, habits, styles, and motivations. Thus, it should therefore be seen as something that can be isolated and studied on its own. However, the fact that actors always act in contexts means that internalized culture always must be brought to 
bear on landscapes equipped with their own brute meanings (see Searle, 1995). Combining these views, sociologists need simply look at both actors and contexts, as well as actors in contexts (see Porpora, 2016). This will prevent sociologists who are more interested in the role that internalized cultural residua play in shaping action from producing either overly deterministic "social hydraulic" accounts (Archer and Elder-Vass, 2012, p. 102; Pugh, 2013) on the one hand, while also preventing sociologists more interested in the situated nature of action from producing overly situationalist accounts (Vaisey, 2009) on the other hand.

In this paper, I argue that while these two views may be complementary, they do not amount to a complete account of how people actually think or act. This is because both views divorce the act of cognition from a cognizing person, and do so using assumptions about the mind as being primarily passive (see Vaisey, 2009) and individuals' decisions as being primarily reactive (see Mills, 1940; Swidler, 1986, 2001; DiMaggio, 1997). Under these assumptions, rather than focusing on how actual individuals think and act, sociologists have increasingly been exploring how internalized cultural elements process information for individuals (see Vaisey, 2009; Calarco, 2014).

While the idea of a passive mind full of mental processors may sound appealing to sociologists interested in implementing policy changes, this idea rests on an untenable model of cognition (see Barkow et al., 1992; Campbell, 2013). Rather than assuming that individuals can be guided predominantly by unmediated, unconsciously held cultural residua, with their conscious faculties kicking in only when their automatic responses fail them (see Vaisey, 2009), recent research has demonstrated the inseparability of one's internalized experiences from one's conscious faculties (Evans, 2010, 2012; Leschziner, 2015). In this latter view, the mind is always actively involved in shaping how one internalizes the world one inhabits. Because of this, any inquiry into the role that cultural residua play in shaping one's actions cannot operate under the assumptions that they are seamlessly internalized and do not vary in meaning across individuals. This means that internalized cultural elements may not be able to be studied in the abstract outside of the individuals who use them within the context of their own lives (see Strauss, 2006).

Since patterns in action do indeed exist, I do not wish for my argument to be interpreted as some kind of chaos theory. Instead, I merely seek to add a level of precision to studies of culture that focus on how cultural elements factor into individuals' everyday perceptions and actions (see Vaisey, 2009; Patterson, 2014). I propose that keeping both mind and environment simultaneously in the analysis enables sociologists to capture what I term active intuition: the process by which individuals spontaneously respond to stimuli using the schematic knowledge that they have acquired in the form of both articulable representations (DiMaggio, 1997) and tacit understandings (Bourdieu, 1984; Vaisey, 2009), as well as the patterned ways of engaging in of automatic self-talk or internal dialogue that they have developed over their lifetimes (see Latinjak et al., 2014, 2016; Van Raalte et al., 2016).
I also introduce the concept of cognitive field to demonstrate how such active intuitions occur within the larger process of schematic shifting and blurring in the decision-making process. As an individual thinks about a given topic, particular memories, assumptions, and mental schemas become "activated" (Cerulo, 2010). Exploring how such activation establishes a cognitive field through which proximate decisions are made by the individual offers a view quite different from the leading perspective of cognitive schemas: it shifts the focus onto how cognitive schemas anchor, frame, and filter one's decisions rather than provide a direct or complete lens through which one can interpret future stimuli. Similarly, it also reframes discussions of the role of reflexivity or conscious decision-making by taking both individual biology and habit formation more seriously than is often the case (see Piiroinen, 2014). Rather than conceiving of reflexivity as a necessarily conscious, deliberate task performed through a process of individuals' abstracting themselves from their social environments (see Archer, 2000), following Decoteau (2016) I argue that reflexivity can be largely tacit and habitual. However, while Decoteau (2016) focuses primarily on reflexivity as being routinized through the development of one's habitus, I emphasize reflexivity's development and use using Mead's (1934) ideas regarding internal dialogue and the internalization of the generalized other combined with Durkheim's (1893/1997) ideas regarding individuals' tacit refraction of stimuli in their environments along with research done by psychologists on athletes (e.g., Latinjak et al., 2016; Van Raalte et al., 2016) and introverts (e.g., Gale, 1983; Johnson et al., 1999) which demonstrate the simultaneously spontaneous and habituated nature of reflexivity.

By combining these lines of inquiry to analyze respondents' own articulations about death and dying, I find that different publicly available schematic understandings of death and dying are used by respondents in patterned ways that challenge both notions of reflexivity as a necessarily conscious act and one emerging out of the interplay of multiple habituses or social positions. Instead, following Wiley's (2016) claims that internal dialogue is an ongoing process of meaning-making that can perhaps be best conceptualized as operating within a larger field of controls, I find that the interview process itself enables individuals to not only drum up ideas that they have about death and dying, but to have their ideas about this topic emerge as they develop their answers and establish a cognitive field. This is evidenced by respondents engaging both in spontaneous deliberation when giving answers as well as in more thorough conscious reflection of what they believe as they verbally state their answers. As such, the argument I put forward follows the lead of scholars of intuition (e.g., Evans, 2010, 2012; Leschziner, 2015) who see intuition as a third way of thinking in addition to conscious and unconscious thought, that is "not driven by deliberation but by intuitions experienced as a sense of what feels right" (Leschziner, 2015, p. 119). Nesting this "feeling of rightness" (Evans, 2012, p. 127) within cognitive fields that emerge in the interview process enables me to foreground the active and dialogical nature of intuition in moments of uncertainty and, as a result, extend this line of thought by specifying how and why automatic schematic understandings become drummed up, 
used, challenged, and mobilized as they are when individuals are responding to questions.

To make my argument, I will first discuss how motivation has been and is currently studied by sociologists. I will then discuss how this line of work has been supported by sociologists' recent use of a model of cognition which views all cognition as either unconscious and automatic, or conscious and deliberative, with the former dominating individuals' thoughts and actions. Drawing largely from work on expertise and decision-making, I will then discuss how this model-the dual-process model (see Lizardo et al., 2016; Moore, 2017) - fails to capture the ways in which individuals engage in everyday deliberative action. To further support the idea that the dual-process model does not accurately portray cognition in practice, I will turn to the work of Emile Durkheim and George H. Mead. Using Durkheim's ideas about how individuals actively refract collective meanings on a continual basis, Mead's ideas about internal dialogue between the "I" and the "Me," and psychologists' claims regarding internal dialogue as an ongoing process, I argue that the sociologist's role is not to capture motivation for action but rather the discrete cultural materials that individuals convert into personal motivations as well as the process by which individuals make such conversions. To show this conversion process in practice, I analyze interviews where individuals make decisions about the life prolongation choices of others. Using my finding that individuals both actively and spontaneously refract collective ideas about dying to make these decisions, I then discuss what implications this has for sociological inquiry.

\section{MOTIVATIONS IN SOCIOLOGY}

After severely diminishing the role that values play in shaping individuals' actions (e.g., Swidler, 1986), many sociologists increasingly turned to structural explanations of action (see Patterson, 2014). Rather than explain the ways in which individuals form lines of action based on values that they have, these sociologists came to place more emphasis on the conditions in which individuals act (Swidler, 1986; DiMaggio, 1997). This shift ultimately changed the conversation from looking at the ways in which culture provides clear guidelines for action to the ways in which it provides opportunities for action (Swidler, 1986; Patterson, 2014).

However, such a shift led other sociologists to question just how fruitful focusing on the situational determinants of action really is (e.g., Vaisey, 2009; Patterson, 2014). While the idea that individuals make decisions based on the opportunities that they have available to them is indeed part of the picture, are values really secondary in -if not totally ousted from-the decisionmaking process? Is the "reification" (Giddens, 1984, p. 180) of ideas in the form of social structures so complete, so strong, that any idea a person has is merely a function of the situations she finds herself in and hence never actually causal (or at least not worthy of sociological analysis)?

Turning to sociological and anthropological work of the structuralist, post-structuralist, and practice traditions (e.g., Bourdieu, 1984; Giddens, 1984), sociologists who were skeptical about the diminishment of values increasingly began to bring values into their analyses through the backdoor. Sometimes they were even brazen enough to bring them in through the front door. Charging in like a proverbial elephant, Vaisey (2009, p. 1676) argued that cultural meanings become deeply internalized by individuals, in turn forming the bases from which they make any and all decisions. According to Vaisey (2009, p. 1676), people do not just act in piecemeal ways, or even according to some larger "strategy of action" (Swidler, 1986, p. 276) that they may have, but in very path-dependent ways based on understandings they may not even know they have.

\section{Dual-Process Model: The Direct Internalization of Schemas and the Production of Homo-Duplex}

To support his claims regarding the path-dependent and unconscious nature of human understanding, Vaisey (2009) used the "dual-process" model of cognition. According to this model, individuals engage in two kinds of thinking: fast, unconscious and automatic thinking, and slow, conscious and deliberative thinking (Vaisey, 2009, p. 1679). Unconscious and automatic thinking consists of tacit pattern recognition, habitual ways of interacting with one's environment, and habitual ways of interpreting stimuli (Vaisey, 2009, p. 1683). Conscious and deliberative thinking consists of purposely thinking about something, attempting to re-frame, re-articulate, or reconceive something that one has perceived, or solving a problem that one does not already know the answer to (Vaisey, 2009, p. 1688). As conscious and deliberative thinking takes a great deal of focus and mental effort, the model holds that individuals will act unconsciously and automatically most of the time. Conscious and deliberate thinking will tend to occur only when unconscious and automatic thinking fail to serve a particular purpose for an individual (Vaisey, 2009, p. 1679). For example, a person may engage in typing on a computer keyboard in an unconscious and automatic way up until the point where she makes a mistake. This can then trigger her to consciously and deliberatively look at the keyboard to figure out where a certain key is located.

If individuals are seen to act automatically most of the time, and this automaticity is indeed going on without any interference from a person's conscious faculties, then culture's influence on action must indeed be quite deep-seated for at least two reasons. First, something needs to be forming these automatic stores if people are not doing it themselves. Second, these automatic stores need to be interpreting or filtering things for the person in ways that allow her to place her conscious mental focus on other things. The power of this logic has led sociologists to increasingly rely on metaphors of transposability (Bourdieu, 1984; Lizardo and Strand, 2010) and internalization to make the case that, once internalized, culture shapes action from the inside out because it has to.

One example of this necessary use of the logic of internalization is the increasing usage of the concept "schema" to explain how various logics of action are present in individuals' broader cultures, which then become internalized by individuals without these individuals cognitively interfering or challenging 
the strictures of these schemas. Schemas are typically defined as mental shortcuts or cognitive processors that individuals internalize throughout their lives (Patterson, 2014, p. 9). For example, Vaisey (2009, p. 1703) argues that "moral-cultural schemas" can explain individuals' actions in ways that cannot be reduced to any conscious articulation because they provide individuals with unconscious logics and motivations for action that may never even be known by one's conscious faculties. One of these schemas was derived from answering the survey question "Do what you think God or scripture tells you is right?" in the affirmative (Vaisey, 2009, p. 1691). Because the affirmative answering of this question was linked to lower levels of deviance in one's future regardless of whether one justified their actions according to this kind of moral schema, Vaisey (2009, p. 1705) concluded that this cultural value could unconsciously steer one away from deviant actions.

Such a view of the driving powers of schemas is similarly found in much psychological work, though schemas here are seen as being more flexible. For instance, Bem (1981) argues that individuals internalize "gender schemas" based on the society they live in. Despite the fact that these schemas can evolve and take on more personalized meanings, they do so in ways that tightly map onto existing norms about what gender is and how one should act as a gendered person (Bem, 1981, p. 354355). Thus, in the West, the reified-structure of a gender binary is accepted automatically by many individuals because they are-quite conveniently-automatically internalizing a model of gender which happens to conform exactly to idea that gender is a binary (Bem, 1981, p. 362-363).

If all schemas operate like Vaisey's moral schemas and Bem's gender schemas, the logic therefore continues that individuals can safely act unconsciously the bulk of the time as they gradually become endowed with schemas for a wide variety of actions, with these schemas accurately modeling the reified-structures or symbolic representations or collective understandings that they are making cognitively possible for these individuals. Whether they are more rigid like Vaisey's (2009), or more flexible like Bem's (1981), the notion that external ideas and logics for action become implanted in one's mind and are fundamentally causative of actions is supported in both views.

\section{Critiques of the Dual-Process View: Intuition and Expertise}

The idea that individuals both internalize schemas in an unmediated way and deploy these schemas unconsciously and automatically to perceive the world around them has been challenged by recent psychological work on intuition. While intuition is often viewed as a strictly unconscious capacity (Vaisey, 2009), Evans (2012) argues that this unconscious capacity is highly dependent on one's experiences. As such, while operating at an unconscious level much of the time in people's everyday actions, it is developed in a way that is irreducible to strictly unconscious means. He argues this based on the fact that despite intuition's unconscious and often unintended impact on how one responds to stimuli in one's environment, intuition is something that is deeply shaped by one's experiences and conscious efforts (Evans, 2010, 2012). In this sense, intuition is more akin to a malleable habit, or a habitual way of seeing, than to a solid, deeply-ingrained mental disposition that one has.

Branches of sociological work on expertise share Evans (2012) focus on the dynamic nature of one's taken for granted, unconscious capacities. In turn, they implicitly work to challenge the view of the mind posed by proponents of the dualprocess model. For example, Vaughan (1996, p. 63) argues that individuals come to develop distinct "worldviews" based on the way they develop skills and proficiencies. A worldview in Vaughan's (1996) sense is very much like an intuition that has been developed in a specific domain to ease future actions by routinizing and automating actions that at one time required a great deal of conscious effort. For example, a butcher learns over time not to associate the pulling out of a knife as dangerous, while a parent would immediately think so upon seeing their children pulling out a knife from the kitchen knife rack (Vaughan, 1996, p. 62). In this sense, the dangerous quality of a knife can be seen as something that changes through conscious and tacit learning.

\section{Durkheim and Mead: The Crafting and Questioning of One's Expertise}

While sociological work on expertise offers one example of empirical support for a view of active intuition or a model that challenges that of the dual-process model, where it lacks explanatory power is in terms of explaining how and why individuals happen to interpret stimuli in the specific ways that they do. If a butcher learns to associate knives with her newly crafted skill with knives, and hence not be afraid of cutting herself with them, does this then mean she is incapable of being afraid of them? If this is the case, then this view of cognition is not dynamic at all. The mind is active up until the point that it crystallizes a new line of automatic thinking, or a new way of interpreting stimuli.

Such questions of turning points (Wheaton and Gotlib, 1997) and identity shifts (Swidler, 2001) have long puzzled sociologists. Two such sociologists whom I believe can fruitfully add support to a view of active intuition are Emile Durkheim and George H. Mead, due to their marked focus on how the stimuli are both interpreted and put to use by individuals. Durkheim's focus on how individuals perceive collective meanings can be combined with Mead's specification of this process as active, ongoing internal dialogue in order to make sense of how a process of selection may be at place when individuals are confronted with stimuli that cannot be reduced to the automatic deployment of schemas. Foregrounding this process of selection, while theorizing it as an unconscious and ongoing process that constitutes a field of its own, provides strong foundations for a view of active intuition.

\section{Durkheim: Collective Representations and Refractions}

Durkheim struggled with the fact that individuals can and do differ in their perceptions of phenomena that can be seen to have shared, historically rooted characteristics. To deal with this paradox, he saw collectively understood phenomena as collective representations and individuals' perceptions of them 
as refractions. For Durkheim (1893/1997, p. 40), collective representations are ideas which have been crystallized into institutions, norms, and laws. These representations are not directly perceived by the individuals who encounter them, however, but are refracted through both individuals' dispositions and personal traits, as well as by the particular situation in which the representation is encountered by any given individual (Durkheim, 1985/1982, p. 37). The meaning that any collective representation has in itself is different than what it has for any particular person based on the fact that it is never actually seen $a$ s such by individuals, but $a$ s it is refracted through myriad elements that a person uses to actually perceive it. In this sense, collective understandings must always be seen as collectively shared but personally understood.

\section{Mead: Internal Dialogue}

The idea that individuals can select from different understandings of any given thing necessitates theories which see meaning-making as an individualized process of selection. Viewing perception and decision-making as highly personalized processes in a way similar to Durkheim, sociologists such as Archer (2007, p. 2) argue that internal dialogue or self-talk must be seen as a routine part of individuals' daily lives which helps people make these selections. Archer (2007) and Archer and Elder-Vass (2012, p. 102) argue that it is only by paying attention to the deliberate ways in which individuals interpret or "activate" the ideas that they hold and the situations they confront that sociologists can understand both how individuals actually form lines of action, as well as what actually motivates them to act or continue acting.

The idea that one can hold ideas which need to be activated by some kind of active self was bolstered by the social behaviorist George H. Mead. Mead (1934, p. 162) viewed action as the interaction of the spontaneous, acting self-what he termed the "I"-and the internalized ideas of others,- - the "Me." Because the self is seen as split in this way, there is always an element of chance in a person's actions because of the distance between the acting "I" and the storehouse of ideas within the "Me" (Mead, 1934; Joas, 1996).

This idea of chance and spontaneity inherent in action by way of internal dialogue necessitates exploring the ways in which individuals convert external meanings or stimuli into personally meaningful representations; it necessitates exploring how the "public" becomes personal, rather than assuming that the "public" automatically becomes assimilated into persons. For instance, bringing these ideas to bear on the idea that individuals can be said to act automatically or unconsciously most of the time (Haidt, 2001; Vaisey, 2009), if the "I" does happen to act largely in accordance with the "Me"-that is, if a person acts in ways which closely mirror collective understandings-this is not because the ideas that one has internalized are completely eclipsing one's capacity to think and act, but rather that these individuals are continually investing these meanings with credibility (Mead, 1934; Archer, 2007). For example, a person can increasingly learn that soccer has fixed rules not because she cannot conceive of soccer any other way, but because she has learned over time that this is simply how others tend to interpret the rules and what will lead her to have fun soccer games. While she could still question these rules, doing so may not yield very much benefit for her, and in turn, lead to the conclusion that she uses this dominant collective understanding of what soccer is and how to play it more often than not. Seeing the halting of questioning soccer's meaning in this sense, then, once again demonstrates how the language of automaticity and deliberation do not quite capture the process that is going on when a person acts either habitually or deliberately; soccer's rules are not questioned because a person simply cannot or chooses not to question them anymore, but because, over time, it becomes less meaningful or useful for her to do so.

\section{Durkheim and Mead: Refracted Internal Dialogue}

While scholars who focus on internal dialogue have been critiqued for minimizing the role of dispositional factors on how individuals form lines of action (Akram, 2013, p. 48), I believe that studying this process can shed great insights into how individuals develop and deploy schemas or worldviews in ways that are irreducible to either purely automatic, unconscious or purely deliberate, conscious modes of thinking and acting. Viewing internal dialogue as both an automatic and deliberate process can help solve both sides of the problem. Rather than see internal dialogue as something individuals consciously engage in only when prior understandings have failed them (see Archer, 2000; Decoteau, 2016), viewed through the lens of Durkheim's refractions and the refractory process that undergirds all perception, internal dialogue can be seen as constitutive of all types of action. Just as schemas are said to unconsciously guide the way individuals perceive their environments, dialogue between one's "I" and one's "Me" should also be seen as an unconscious guide.

Unconscious or spontaneous internal dialogue has indeed been found to be prevalent among two rather different groups of individuals: athletes (see Latinjak et al., 2014, 2016; Van Raalte et al., 2016) and introverts (see Gale, 1983; Johnson et al., 1999). Athletes have been found to engage in self-talk while engaging in athletic performance both deliberately and automatically (Latinjak et al., 2014), and to do so to either improve or maintain their current performance. For example, Latinjak et al. (2014) found that athletes deliberately engage in positive self-talk, such as telling themselves "you can do it" when they are not performing as they would like. However, Van Raalte et al. (2016) found that athletes spontaneously engage in selftalk when performing regardless of performance. For example, a sample of dart-throwers who were being audio-recorded while playing darts were found to engage in self-talk at multiple periods of their performances (Van Raalte et al., 2016).

Similarly, Johnson et al. (1999) found that there may be evidence that introverts carry out ongoing internal monologs even without the presence of external stimulation. Johnson et al. (1999) sought to isolate casual or unprompted thinking states in introverts and extraverts by testing the cerebral flow levels of these individuals while they were asked to think freely. Introverts were found to have higher levels of activity in the frontal lobe regions of the brain, areas that are used when planning, thinking, and remembering. As a result, the authors inferred that introverts 
engage in internal dialogue automatically at a higher degree than do extraverts.

The idea that individuals engage in self-talk automatically rather than strictly deliberately helps make sense of how individuals can come to engage in seemingly reflexive or deliberate action without necessarily having conscious or deliberate intent (see Kahneman, 2011; Leschziner, 2015; Decoteau, 2016). Seeing schemas as taking on their precise shape in situ helps make sense of this. When a butcher sees a knife, this activates her ideas about knives being either dangerous weapons or cutting tools (see Leschziner and Green, 2013). In order for this activation to occur, she has to assess the context she is: is she in a kitchen or elsewhere? If she is indeed an expert butcher, this assessment may happen unconsciously, as over time she would naturally come to stop thinking about the danger that the knife represents to her at work (see Leschziner, 2015). Should the butcher come to fear the knife within this context and feel silly for doing so-imagine a case of "What was I thinking?"-this is not because she has not developed the expertise or wherewithal to automatically know that knives are not dangerous here, but rather that her "I" mucked up the situation by selecting the wrong collective representation of knives from her repertoire of understandings. This mucking up of the process of selection of one's schemas by the "I" signals that the process of internal dialogue is indeed going on, and that, since she did not even realize why it happened this way-she thought it was a solid habit or routine-that it is a dialogue that is going on automatically and unconsciously.

In this sense, her interpretation of the knife as dangerous in the context of the kitchen can be seen as not only a mishap or muck up, but, similarly to the dart-throwers who spontaneously engaged in different kinds of self-talk when performing and the introverts who automatically engage in self-talk when tasked to think freely, as indicative of the patterned spontaneity of action, thought, and perception. If the interpretation and, in turn, reaction to a knife can be reduced to an automatized unconscious process of internal dialogue between one's active "I" and one's acquired sets of knowledge, then it must also be reducible to the idea that this moment of interpretation can be impacted-by a person calling upon a different set of knowledge than what they usually would, such as the collective representation that "knives are dangerous"-and, therefore, that this impact has both elements of pattern and spontaneity to it. If the butcher fears the knife when she sees it in the kitchen, this may be a chance mistake, but it is one that is happening because of the particular sets of knowledge that she has: knives as dangerous, knives as cutting tools, her own skill with knives, her role as protector of her child, and so on. In short, both her tendency to views knives a certain way and to not view them in other ways cannot be reduced to these modes of perception. Without seeing this dialogue as an ongoing process of selection between modes of perception, mistakes would be relegated to some kind of schematic failure rather than seen as the product of having a mind full of many schemas, ideas, and understandings which could possibly influence how one refracts something that confronts oneself. Similarly, dart-throwers spontaneous instances of self-talk would be deemed as more deliberate and crafted than they may actually be.

\section{DEATH AND DYING: PERSONALIZING NOVELTY}

Due to massive technological innovations throughout the Twentieth century in much of the world, individuals who become terminally ill now often face the dilemma of having to decide whether they will have their lives prolonged (Seymour, 2003). As the opportunity to prolong one's life is a fundamentally personal dilemma, I believe exploring the ways in which individuals think about making this decision can enrich our understanding of how tacit understandings and ideas about the world shape novel decisions that individuals make-or even decisions that they simply entertain. In fact, this subject's abstract quality is methodologically beneficial for this project since it helps make explicit the ways in which individuals personally endow meaning to collective representations or schemas in situations in which no single automatic way of responding is widespread-there is no simple way to think about or respond to questions about life prolongation due to its blurry status, therefore necessitating that respondents engage in reflexive deliberation, spontaneous use of loosely defined understandings, or a combination of the two.

Moderate patterns in making sense of this turning point in one's life have been found by numerous studies, however. Some patterns of sense-making are the following considerations made by individuals: How much of a burden one may be to their loved ones should one decide to have one's life prolonged (Seymour, 2003, p. 339; Singer et al., 1999, p. 166); how effective life prolonging strategies actually are (Singer et al., 1999); the level of comfort one is in Braun and Zir (2001, p. 693); and whether life after life prolongation will be worth living (Vandrevala et al., 2006). Studies have shown that the use of these factors or schemas for understanding life prolongation at the end-of-life depend on the experiences a person has had with this phenomenon (Vandrevala et al., 2006, p. 1582), as well as their level of knowledge about these procedures (Schonwetter et al., 1993, p. 299).

While establishing that these concerns are common among many individuals is useful for shaping policies directed at endof-life care, they are less useful for telling us about how and why these concerns are so salient in particular individuals' ideas about life prolongation. While studies often connect a particular concern to a particular institutional history in the country or city in which an individual lives - for instance, stating that living in an "individualist" country such as Britain can lead to individuals focusing on their own personal well-being at the end-of-life (Broom and Kirby, 2013) - the process by which such decisions are made are little known. As such, as I will discuss below, my focus is less on the final conclusions that individuals make, and more on the routes they take to get to these decisions. Examining such routes will enable me to demonstrate the way individuals actively intuit ideas about life prolongation in the interview setting. In extension, it will serve as a case study for how individuals may actively intuit other aspects of their lives 
which are not clearly reducible to either deliberate reflection or automatic processing as these functions are usually depicted.

\section{MATERIALS AND METHODS}

To examine the ways in which individuals refract schemas when making decisions I use a secondary data set which includes in-depth interviews asking respondents about other individuals' decisions to prolong their lives. The data I am using is a set of 39 in-depth interviews conducted by Jane Seymour and Gary Bellamy. Data collection took place from 2001 to 2002 in Sheffield, UK. The original study was carried out in accordance with the recommendations of the British Sociological Association, and received ethics approval from the North Sheffield Research Ethics Committee. All subjects gave written informed consent in accordance with the North Sheffield Research Ethics Committee.

Respondents are stratified by age (65-74, 75-85, and 85 and over), gender, neighborhood deprivation (high-, middle-, and low-deprived neighborhood), and living arrangement (own home or in a state facility). All respondents identify as "White British" and as being healthy (not chronically or terminally ill), and were randomly sampled by a series of general practitioners in the Sheffield area who had many respondents to sample from.

In these interviews, respondents were given vignettes of two fictional individuals-Frank, a man with terminal lung cancer; and Margaret, a woman with Alzheimer's-who gradually become more ill throughout their respective narratives. They are first asked a series of questions about how Frank and Margaret might react to doctors' suggestions of using particular medical devices to prolong their lives. They are shown pictures of the devices and are told how and why they would be used to prolong either Frank or Margaret's life. The interview then moves onto questions involving these characters ultimately making the final decision to either prolong their lives or to die "naturally" after having considered these various life prolongation tools and strategies. Based on the information given about these characters, respondents are asked whether they think that Frank and Margaret themselves would want to prolong their lives. For the purposes of this study, I will be focusing on Frank.

The use of hypothetical scenarios in which dying respondents must choose whether or not to prolong their lives carries a series of benefits. First, respondents are able to place themselves in the hypothetical character's situation. This enables respondents to think about death and dying in relatively concrete terms, thereby enabling researchers to capture a simulation of the decision-making process that individuals might make if they were faced with their own deaths (Seymour, 2003, p. 341). The use of vignettes has been done for similar purposes by sociologists such as Ann Swidler (2001). Swidler (2001) used vignettes about married individuals in an effort to elicit tacit assumptions that individuals might make when making decisions for themselves but would feel the need to justify when making these choices for others. This added specificity to one's answer will help me establish just how and why certain schemas apply to a respondent's answer and how and why others do not. For instance, if a respondent were to state that Frank would likely want to end his life due to being uncomfortable, the fact that they are making this decision on Frank's behalf may prompt the respondent to give additional explanation for why exactly this may be the case.

Such added justification will also likely cause respondents to engage in more rigorous self-talk. This is because they may feel the need to put themselves firmly in the other's shoes to make this kind of decision. While individuals always do this to an extent when making decisions for themselves, making decisions for others can prompt individuals to think more deeply about the tacit assumptions that might guide their decisions (Swidler, 2001).

Given the emphasis I am placing on internal dialogue, putting oneself in another's shoes, and justifications of one's answers, I will be approaching the interviews using the approach of narrative analysis (Hollway and Jefferson, 1997). In this approach, rather than mining the interview transcripts for nuggets of information, researchers tend to be interested in the decision-making processes respondents engage in and the ways in which these processes take specific shapes as trajectories (Hollway and Jefferson, 1997). In my case, I will be focusing on which schemas are used at which time in the interview, how the use of these schemas change throughout the interview, and how different final conclusions to questions vary based on which schemas are used early in the interview process as cognitive "anchors" for the decision-making process and trajectories respondents embark upon (see Berg, 2014, p. 1). Doing so will enable me to bypass one of the major limitations of the data I am using: a lack of knowledge regarding much of the respondents' lives both prior to and outside of the interview process.

\section{RESULTS}

When asked whether "Frank" would opt to prolong his life after experiencing serious bouts of breathlessness and knowing that he is terminally ill with cancer, respondents tended to loosely develop their responses in three tiers:

1) Consultation: In the consultation stage, respondents would discuss how Frank should be made aware of the resuscitation options available to him. However, if he is not mentally capable of understanding the procedures or the severity of his situation, then his wife should be told instead.

2) Comfort: Once asked whether Frank would actually want to prolong his life after a consultation, respondents tended to initially frame their answers in terms of how much pain Frank was in. If Frank is not in a great deal of pain, then he would likely opt for life prolongation. However, if he is comfortable but thinks that he might fall ill again in the future, he may reconsider his decision to prolong his life.

3) The Value of Life: After discussing comfort, respondents would then tend to either affirm or challenge their assumptions about Frank by discussing how the meaning of one's life is intimately personal. It would make sense that he considers the amount of pain he is in as well as how 
"livable" his life will be after life prolongation, but perhaps he may evaluate things that one cannot foresee without actually knowing him.

Respondents tended to answer the question using all three of these levels of response in the order presented here, though in different ways. In the following, I will review each step and demonstrate how respondents made sense of Frank's choices in personal yet patterned ways. I am focusing on only a handful of respondents to keep the analysis relatively brief. I attempted to capture the variety of responses that I found by analyzing the responses of interviewees that were typical of the sample, but have also emphasized some who were particularly unusual or eloquent in their responses. Doing so enabled me to showcase the variety the ways in which respondents used schematic understandings of death and dying.

\section{Step 1: Consultation}

In this first step, when asked whether it is "right" for doctors to discuss resuscitation with Frank at all, many respondents would put themselves in Frank's place by considering how his level of physical, emotional, and psychological wellness would weigh in on his decision to prolong his life. For example, Eddy said that such a discussion should only take place if Frank is "fit enough and he knows what he's saying." However, upon further reflection, he then went on to say that "if his mind's gone it's pointless talking to him." For Eddy, then, the discussion of life prolongation with Frank at this point of his life was fine as long as Frank could actually understand what the doctor was saying. To make this decision, Eddy first drummed up ideas about being "fit enough" to understand questions addressed to oneself, and then further concretized this statement by stating how talking to individuals who have lost their minds is pointless.

However, other respondents felt that this discussion was too important to occur if Frank was just sentient "enough" to partake in the conversation. For example, Larry said that Frank is "far gone now" and that "they should just let him go." Larry then went on to say that "well if he's sane enough and his mind's lucid enough, they talk to him, there's that way. But if he's not, I think it's a waste of time." However, to justify his initial claim that Frank was too "far gone" to be discussing this, he explained the situation of a friend of his whose experience with breaking her leg and having it fixed caused her to suffer a great deal. Using this example, he explained that the severity of the decision to prolong one's life is so extreme that he personally would not want to do it as he "wouldn't want to be brought back again to be in the same position as I were before it happened."

Mary echoed Larry's hesitation toward the idea that Frank should be discussing life prolongation at this time of his life. According to Mary, someone who has fallen ill is not in a place to be making such a grave decision:

Are you in a position to make such a mind-blowing decision like that when you're having breathing difficulties in hospital? Are in you a position to discuss it with a doctor? Are you? It's like saying do you want to live or do you want to die? I can't envisage a question like that, I can't.
Mary then went on to say that a person should make decisions about life prolongation well before they wind up very ill, so that they can make a more carefully planned decision. Were she to become terminally ill herself, however, she is sure she would not want to be resuscitated:

Well, prolonging something that's wanting to be ... out of it ... anyway-I can't see the point of. If you had an animal who was suffering, you wouldn't want that, would you? [chuckles] While you wouldn't give the terminating needle to a human being, like you would to an animal, erm, you wouldn't be resuscitating that dog and keeping it going while it was suffering, would you?

Overall, when addressing the question of whether Frank should discuss resuscitation after his bout with breathlessness, respondents tended to agree that this was ultimately Frank's decision, but framed their answers around their own feelings about what they themselves would do should they fall ill. This process tended to begin with the notion that if one is sentient enough to understand the gravity of the issue, then one should be told what one's life prolongation options are. However, upon saying this-or activating what I call the "sentient" schemaindividuals then refracted these ideas into a particular stance on the issue. The expansion of this schema into the use of evidence either in favor of or against the idea that Frank should discuss life prolongation strategies thus appeared as a common response pattern in how respondents initially answered questions about Frank and his dilemma. At this point in the interview process, respondents were just beginning their internal-as well as their explicitly intersubjective-dialogues about how one may go about making this kind of decision. Emergent ideas began to come out more once comfort, well-being, and autonomy came into the picture.

\section{Step 2.A. Comfort}

While most respondents agreed that life prolonging strategies should be discussed with Frank, respondents began to more markedly vary in terms of whether or not Frank should (or would) actually opt for the use of these strategies. The first criterion that tended to emerge was that of comfort. Should Frank be in a state of minimal pain, then he perhaps would likely want to have his life prolonged. However, if he is experiencing a significant amount of pain, he might opt out of this process. Respondents tended to discuss issues of comfort after making firm claims about whether Frank should or should not prolong his life.

For example, Linda stated that "even though he's so ill, I suppose you want to go on as long as possible." However, she then augmented this claim by stating this would be so "unless he was in too much discomfort." Similarly, Mike claimed that his philosophy is that "where there's life, there's hope." $\mathrm{He}$ then went on to say that Frank would likely want to keep on living, implying that this is just what people do. However, Mike soon augmented this claim and appeared to challenge his own worldview by saying that Frank's decision rests on how much pain he is experiencing. While Mike likes to think that he himself will be optimistic enough to undergo life prolongation should the need arise, he understands that "it's really up to the patient" and that he "suppose[s] how much pain he is going through 
[influences] whether he wants it." What Mike thinks Frank, or anyone else, would actually do is unclear at this point. What is clearer, however, is that despite Mike's self-proclaimed guiding ideology about willful living, he can and does see that multiple factors may be considered in this choice not only by other people, but potentially by him as well.

One respondent who would fit Mike's description of how one might make this decision is Linda. When asked whether she thought it was natural to prolong one's life at all costs, she stated that:

I think if your time has come and you know it has, then no don't try to prolong it, 'cos it, I would imagine that you go through more pain and more discomfort; you're putting your family through more as well. I think myself, if it was me, I would say, no, don't do it.

While Linda ultimately viewed comfort as a criterion that she must meet in order to prolong her own life, Mike saw this as being important primarily for others. For Mike, then, issues of discomfort came up to justify deviations from his overall view of the situation: Life should be prolonged, unless one is in "too much" pain. In other words, issues of comfort did not really factor into the decision he made regarding life prolongation, but did factor into what he thought others-such as Frankmight consider should they be facing life prolongation. Comfort as a necessary criterion for prolonging one's life, then, served as a collective representation in responding to the question of whether Frank would prolong his life for both Mike and Linda in that they both saw it as an important criterion to consider, but actually functioned as a refraction for both of them: comfort did not matter in light of Mike's larger life philosophy, but it did for Linda. In other words, Mike and Linda both shared knowledge of comfort as a "public code" that factors into individuals decisions (Swidler, 2001), but differed in regards to the particular meaning it had for him and her personally. The meaning of this code took on different shapes as it was framed within their particular life contexts, all the while being anchored within the larger social environment which they were a part of that emphasized personal choice and one's right to make this kind of decision for oneself. The processes by which they came to these answers further exposes the ways in which they can and do refract common understandings into personal thoughts.

\section{Step 2.b: Well-Being}

After discussing aspects of comfort that applied to Frank's situation, respondents then often claimed that Frank's decision to be resuscitated hinged on whether his life after this process would be "worth living." While being comfortable is important, one should also consider how likely one is to relapse after this process and endure the trauma of perpetually being in and out of the hospital.

An example of this kind of reasoning was used by Mary, who, as I noted earlier, claimed that life should not be prolonged if one is in a great deal of suffering. After making these claims about comfort factoring into this kind of decision, she then went on to say "I've always enjoyed my health and strength so much that if I have been ill I've thought, 'Oh, I wouldn't want to carry on like that."' Being comfortable is not enough for Mary given the value she gives strength and vitality in her life. However, she hedges this claim by stating "everybody's different. Some people will cling on to life to the Nth degree, won't they?"

Just as Mike could see that comfort factors in to one's decision to prolong her or his life in general, Mary sees that the desire to "cling on to life" also factors into this process. Like Mike, however, this is not the case for her. Given her larger valuation of health and vitality, life should not be prolonged in her case should she become terminally ill. Like Mike, Mary is able to distance herself from the schemas that she thinks might use to consider this kind of decision and assess her own, personal view of both the overall question of life prolongation as well as the use of this particular criterion to make this decision.

\section{Step 3: The Value of Life}

Despite all discussing comfort and well-being to a greater or lesser degree, all respondents ultimately claimed that the decision to prolong one's life was one which came down to a person's overall evaluation of the value of his or own life. While some respondents made claims about what Frank should do, these claims were made using factors that they imagined to be Frank would actually weigh when making this choice. For instance, Barbara claimed that Frank should not opt to have his life prolonged due to the fact that doctors "never really know" whether they will actually be able to save their patients. Coupled with this issue of the uncertainty of medical expertise, Barbara also claimed that doctors sometimes knowingly persuade patients into having their lives prolonged even when they "know the strategies won't work." This latter claim was reinforced by Barbara's discussion of her sister-in-law and her battle with sudden illness. "The doctors knew she would not get better," yet they "treated her anyway." "They put her through hell, and that isn't right." Because of what happened to her sister-in-law, Barbara believed that it would not be in Frank's best interest to prolong his life. These strategies simply are not guaranteed to work. Furthermore, having seen her sister-in-law buy in to what her doctors told her, she apparently felt impelled to steer Frank in a more informed direction. After further deliberation, however, Barbara's suggestion that he should not prolong his life was hedged by the realization that "only he knows" what his situation is, and that ultimately it is up to him. Still, if "it were happening to me," she said, she would definitely not opt to have her life prolonged.

\section{DISCUSSION}

Looking at the ways in which respondents formed answers using widely available schemas, I argue that my case supports the idea that active intuition constitutes a third alternative to unconscious and conscious thought as they are typically seen (see Evans, 2012; Leschziner, 2015). For some respondents, comfort and well-being are factors that they understand as being important in the abstract, or for other people, but not important to them personally. This is because comfort and well-being are seen personally as less meaningful to these respondents than other values that they hold. As such, comfort and well-being do not hold the same meaning for all individuals. Instead, their 
meanings emerge out of the person's larger set of beliefs and understandings and how these general representations fit into this personalized context (see Leschziner, 2015). Respondents refracted ideas about comfort and well-being that they held in an abstract sense through myriad factors such as life experiences and life philosophies.

Based on widespread understandings of life and death available to individuals living in Sheffield, respondents answered questions about life prolongation automatically using similar themes. However, the deployment of these themes took on different shapes based on both the life history of the respondent and the specific trajectory that the interview had taken. In this sense, the deployment of schemas could be seen as an emergent property of the interview, or what many qualitative researchers may call "context effects" (see Burawoy, 1998). However, taking this emergent aspect of the deployment of schemas within interview contexts outside of this context and seeing it as a general property of schemas is something I believe sociologists can fruitfully do. Using the more psychological interpretation of schemas-that they are flexible heuristics that are more akin to habits or other adaptive traits than to fixed rule-sets-one can infer that the development of schemas would be influenced by the contexts in which they are both attained and used (Dewey, 1922/1988; Leschziner, 2015). Schemas, then, may begin relatively fixed and categorizable-the way comfort and wellbeing did in the interviews presented here-but then become more personalized as they are used in the context of a living person equipped with other ideas, nested within a context of action with requirements and impelling forces of its own. The same may be said for collective representations or any kind of publicly available understanding ${ }^{1}$.

The implications of this view of schemas as taking on meaning in situ may change the way sociologists view both schemas and the process of internalization of schemas more generally. While individuals do indeed internalize understandings and skills as they engage in various practices, they do so not by simply absorbing them like sponges, but by refracting them through myriad factors (Durkheim, 1985/1982; Decoteau, 2016). These factors consist of what one has already learned, the habits one has, the field in which these understandings or skills are introduced (Decoteau, 2016), one's current mood (Silver, 2011), and even by what happens to pop into one's head at the moment of perception (Mead, 1934), and the extent to which one engages in reflexive deliberation (Archer, 2007). Because understandings and skills are filtered in this way before becoming internalized, this means that the actual meaning that they bear for a person cannot be reduced to any meaning they have either in the abstract or within a larger community (see Decoteau, 2016).

\footnotetext{
${ }^{1}$ The inference I am making here must be taken with some caution, however, as individuals do not consciously think about all of the automatic decisions that they make (see Vaisey, 2009; Kahneman, 2011). The data I am analyzing gave me access to schematic understandings of death and dying which were used in the context of an interview. As such, respondents were probed and asked to expand on their answers. Without such probing, these ideas may not have been forced into consciousness and thus made actively intuitable - they may have simply given answers and not thought much about them after the fact.
}

If general or abstract meanings take on some of their meanings as they are internalized by individuals, this means that an interpretive process is going on within individuals. For instance, if the abstract notion of "well-being" is given weight in the decision to end one's life for one person but not for another, this signals that the meaning that this shared meaning connotes is importantly personal. As Durkheim (1893/1997) posited, myriad factors may be playing into this attribution. Throughout my analysis, I found that the trajectory the interview was taking was one such factor. Abstract notions of comfort, well-being, and personal choice took on specific values as they were used to understand specific instances of life prolongation and dying. For these individuals, then, tacit understandings were therefore not simply static and waiting to be activated, but took on very different meanings based on precisely how they were activated. Because of this, this activation is not akin to the flip of a switch, but rather, the introduction of a new substance into a chemical mixture or a living organism. It is an interaction and, as such, cannot be understood meaningfully without paying due attention to what actually interacted with it at the time of its emergence.

The agency involved in activating tacit understandings is therefore very different than how it is usually considered. It is neither reducible to effort (see Silver, 2011) on the more agentic side which sees an actor picking and choosing meanings, nor to dispositions (Bourdieu, 1984) on the more structuralist side which pictures a habit that a person has developed somehow converting external meanings into personal meanings via modes of perception that have been built up in a person for that person on its own. While efforts to blend these positions have been fruitfully attempted by scholars such as Decoteau (2016) and Elder-Vass (2010) who have pointed out the fact that reflexive deliberation is highly shaped by both one's prior experiences and one's current context, this process has still been seen as a largely conscious activity. Psychologists' finding about selftalk often being automatic and spontaneous (e.g., Van Raalte et al., 2016) lends plausibility to claims made throughout history by numerous historical figures regarding the role of self-talk in shaping individual perception and, in turn, to my own claims regarding reflexivity as unconsciously motivating action.

For example, in Leviathan (Hobbes, 1914, p. 15) Thomas Hobbes asserted that all perceptions occur within the context of an ongoing "train of thought." He believed that all concepts are interpreted in light of what an individual has already thought as well as on what he is currently thinking, necessitating examination of the moment of perception rather than either the object of perception or the perceiver divorced from the object of perception. Similarly, novelists Virginia Woolf and James Joyce framed many of their characters as being deeply engulfed in their own internal conversations while simultaneously being involved in interactions with others (Auerbach, 1953), leading to the possibility that individuals may be occupying their own cognitive context while occupying particular field positions (e.g., Decoteau, 2016) or other kinds of contexts. Such a possibility shifts attention away from discrete fields that individuals operate in, to cognitive fields that they tacitly construct and enter into as 
they interact both with themselves and with others. In this sense, fields can be conceived as emergent properties of individuals that come to structure their future actions rather than as preexisting residues of social action which exist outside of them (see Lewin, 1936, 1939; Parlett, 1991; Martin, 2003; Wiley, 2016). Seeing fields as sometimes being cognitive accomplishments of dialogue between an individual's "I" and "Me" or as the accomplishments of two interacting individuals helps make sense of how individuals enter into contexts already in context-they enter situations with a train of thought or with some thoughts already triggered, which then filter the perceptions they have in the present moment. Seeing actors as potentially already being in situ reframes the way one can analyze and interpret moments of reflexive action that actors appear to engage in by forcing the analyst to consider just what kind of cognitive field actors have established when giving responses to questions or behaving in particular ways. Rather than simply choosing to be reflexive in any given moment, actors may be impelled into a state of reflexivity by spontaneous connections they make between their understandings or conscious conversations with themselves or others. Thus, while actors may still make reflexive, conscious choices, the motivations behind these choices-or this seemingly spontaneous activity-may be more dependent on individuals' previous actions and interpretations than is often assumed.

Taking these claims about internal dialogue's ongoing and often unintended nature into consideration, I argue that the interviews I analyzed can be utilized to understand how this process involves the use of schemas. As seen in my analysis of interviews and the numerous decision-making pathways respondents took, all external stimuli were refracted by the acting individual who not only is already in possession of myriad internalizations which serve as a context for how any given thing is internalized in the future (e.g., experiences with death and dying one has had, prior learning about comfort and wellbeing, and so on), but who is also occupying a context which endows both all of these internalizations as well as the process of internalization with particular meanings (the interview, the cognitive fields the interview takes people into, and so on) and somehow selecting between various ways of perceiving (respondents beginning discussions either with comfort or with well-being, and then muddling their way through various understandings of these schemas) based on how their more personal context and their more external, situational context interact for him or her.

The impact of the situational context on a person's interpretive process is made clear in Eliasoph and Lichterman's (2003) work on how group styles filter the ways in which individuals draw on collective representations. Through ethnographic observation of a group of bar patrons and a group of suburban activists, they argue that understanding the "culture" of each group is not enough to grasp how the codes and meanings they share are actually mobilized by these individuals. Instead, sociologists must see how these meanings are used "in interaction," as doing so led the authors to find that these groups often demonstrated ambivalence about topics that would not be captured through more de-contextualized analysis of the collective representations or shared cultural meanings they tended to draw on as groups (Eliasoph and Lichterman, 2003, p. 783).

While Eliasoph and Lichterman (2003) focus on the grouplevel and emphasize the importance of seeing collective representations due to the myriad ways in which they are alternated between and mobilized by groups based on their tone or style, the personal ways in which individuals as individuals engage in this meaning-making process is less attended to. The concepts of active intuition and cognitive field enable sociologists to extend Eliasoph and Lichterman's (2003) claims about the weight of context on deliberation to the individuallevel by conveying how self-talk or conversation of oneself has much in common with conversations with others in dyadic or group settings. Moreover, it helps clarify the role that habit and routine play in shaping the use of collective representations by shifting the conversation-literally-to the individual-level where habits are formed and away from more relatively autonomous group settings. For example, individuals with stigmatized identities may come to cycle through schematic understandings of their identities even outside of the confines of conversations or other group situations (Williams, 2017). Williams (2017) found that individuals who self-identified as pedophiles would shift between widely shared understandings of pedophilia-ranging from a sexual orientation to a choiceboth while in conversations with others and more autonomously provoked cries for help posted on Web Forums. Similarly, individuals who faced several career setbacks would alternate between seeing themselves as worthy of gainful employment and as personally responsible for their career failures (Williams, 2018). In both instances, individuals appeared to refract collective representations in ways which were shaped not just by group styles-of pedophiles or individuals with turbulent careers, respectively-but based on the particular outlooks they had developed and which became habitual (Williams, 2017, 2018). Moreover, in both cases such refractions occurred both when directly probed by others as well as in more autonomous, self-prompted ways (Williams, 2017, 2018), signaling that individuals' particular refractory processes themselves can become habitual.

Seeing the spontaneous development of cognitive fields in the interview setting allows us to better see the import of the broader refractory process that individuals engage in when they use collective representations. Seeing this process as mediating at least some of a person's interactions with his or her environment as well as with him- or herself (see Mead, 1934; Lewin, 1936) enables social scientists to study collective understandings in both their discrete forms as objective social facts that these individuals engage with and endow with their own subjective meanings (Durkheim, 1985/1982), as well as to study these facts' acquisition or internalization by individuals in patterned yet emergent contexts. Future studies can focus on seeing internalization of schemas or any form of external culture as scaffolded both by the larger person that one is and has become as well as on their context of use in order to better specify just how and why any given person actually behaves in the ways he or she does. Doing so will place emphasis 
back on individuals in a way that does not reduce action and thought to chaos, but will enable further specification of why certain behaviors tend to be commonplace despite the active and idiosyncratic nature of meaning-making and perception; if we all have the capacity and impulsion to spin narratives and refract stimuli through our self-concepts (see Markus and Wurf, 1987), convergence to common themes and reliance on shared principles becomes all the more interesting (see Kahneman, 2011). Specifying the extent to which one actively intuits aspects of one's environment rather than relying on either a) mostly unconscious, unreflected understandings on the one hand or deeply conscious yet identity non-dependent understandings on the other (see Vaisey, 2009, 2014) or b) situational and

\section{REFERENCES}

Akram, S. (2013). Fully unconscious and prone to habit: the characteristics of agency in the structure and agency dialectic. J. Theory Soc. Behav. 43, 45-65. doi: $10.1111 /$ jtsb.12002

Archer, M. S. (2000). Being Human: The Problem of Agency. Cambridge: Cambridge University Press.

Archer, M. S. (2007). Making Our Way Through the World: Human Reflexivity and Social Mobility. Cambridge: Cambridge University Press.

Archer, M. S., and Elder-Vass, D. (2012). Cultural system or norm circles? An exchange. Eur. J. Soc. Theory 15, 93-115. doi: 10.1177/13684310114 23592

Auerbach, E. (1953). Mimesis: The Representation of Reality in Western Literature. Princeton: Princeton University Press.

Barkow, J. H., Cosmides, L., and Tooby, J. (1992). The Adapted Mind: Evolutionary Psychology and the Generation of Culture. New York, NY: Oxford University Press.

Bem, S. L. (1981). Gender schema theory: a cognitive account of sex typing. Psychol. Rev. 88, 354-364. doi: 10.1037/0033-295X.88.4.354

Berg, J. M. (2014). The primal mark: how the beginning shapes the end in the development of creative ideas. Organ. Behav. Hum. Decis. Process. 125, 1-17. doi: 10.1016/j.obhdp.2014.06.001

Bourdieu, P. (1984). Distinction: A Social Critique of the Judgment of Taste. Cambridge, MA: Harvard University Press.

Bourgois, P., and Schonberg, J. (2009). Righteous Dopefiend. Los Angeles, CA: University of California Press.

Braun, K. L., and Zir, A. (2001). Roles for the church in improving end-oflife care: perceptions of christian clergy and laity. Death Stud. 25, 685-704. doi: 10.1080/713769897

Broom, A., and Kirby, E. (2013). The end of life and the family: hospice patients' views on dying as relational. Sociol. Health Illn. 35, 1-15. doi: 10.1111/j.1467-9566.2012.01497.x

Burawoy, M. (1998). The extended case method. Sociol. Theory 16, 4-33. doi: $10.1111 / 0735-2751.00040$

Burt, R. S. (1982). Toward a Structural Theory of Action: Network Models of Social Structure. London: Academic Press.

Calarco, J. M. (2014). The inconsistent curriculum: Cultural tool kits and student interpretations of ambiguous expectations. Soc. Psychol. Q. 77, 185-209. doi: $10.1177 / 0190272514521438$

Campbell, A. (2013). A Mind of Her Own: The Evolutionary Psychology of Women. Oxford: Oxford University Press.

Cerulo, K. (2010). Mining the intersections of cognitive sociology and neuroscience. Poetics 38, 115-132. doi: 10.1016/j.poetic.2009.11.005

Cohen, M. D., March, J. G., and Olsen, J. P. (1972). A garbage can model of organizational choice. Adm. Sci. Q. 17, 1-25. doi: 10.2307/23 92088

Decoteau, C. L. (2016). The reflexive habitus: critical realist and bourdieusian social action. Eur. J. Soc. Theory 19, 1-19. doi: 10.1177/13684310155 90700 group constraints which delimit how decision-making can take place (see Cohen et al., 1972; Janis, 1972; Burt, 1982) will enable better clearer discernment of how and why culture motivates and justifies actions as it does. It will do so by seeing the possibility of active intuition, or of the personalization of collectively shared understandings, as a potential response to the myriad situations individuals confront on an everyday basis.

\section{AUTHOR CONTRIBUTIONS}

The author confirms being the sole contributor of this work and has approved it for publication.

Dewey, J. (1922/1988). John Dewey, the Middle Works, 1899-1924: Human Nature and Conduct, Vol. 12. Carbondale, IL: Southern Illinois University Press.

DiMaggio, P. (1997). Culture and cognition. Annu. Rev. Sociol. 23, 263-287. doi: 10.1146/annurev.soc.23.1.263

Durkheim, E. (1893/1997). The Division of Labor in Society. New York, NY: The Free Press.

Durkheim, E. (1985/1982). The Rules of Sociological Method and Selected Texts on Sociology and Its Method. ed S. Lukes. New York, NY; Toronto, ON: The Free Press.

Elder-Vass, D. (2010). The Causal Power of Social Structures: Emergence, Structure and Agency. Cambridge: Cambridge University Press.

Eliasoph, N., and Lichterman, P. (2003). Culture in interaction. Am. J. Sociol. 108, 735-794. doi: 10.1086/367920

Evans, J. S. B. T. (2010). Intuition and reasoning: a dual-process perspective. Psychol. Inq. 21, 313-326. doi: 10.1080/1047840X.2010.521057

Evans, J. S. B. T. (2012). Spot the difference: distinguishing between two kinds of processing. Mind Soc. 11, 121-131. doi: 10.1007/s11299-012-0104-2

Gale, A. (1983). Electroencephalographic studies of extraversion-introversion: a case study in the psychophysiology of individual differences. Pers. Individ. Differ. 4, 371-380. doi: 10.1016/0191-8869(83)90002-8

Giddens, A. (1984). The Constitution of Society. Berkeley: University of California Press.

Haidt, J. (2001). The emotional dog and its rational tail: a social intuitionist approach to moral judgment. Psychol. Rev. 108, 814-834. doi: 10.1037/0033-295X.108.4.814

Hobbes, T. (1914). Leviathan. London: Dent.

Hollway, W., and Jefferson, T. (1997). Eliciting narrative through the in-depth interview. Qual. Inq. 3, 53-70. doi: 10.1177/1077800497003 00103

Janis, I. L. (1972). Victims of Groupthink. Boston: Houghton Mifflin.

Joas, H. (1996). The Creativity of Action. Chicago, IL: The University of Chicago Press.

Johnson, D. L., Wiebe, J. S., Gold, S. M., Andreasen, N. C., Hichwa, R. D., Watkins, G. L., et al. (1999). Cerebral blood flow and personality: a positron emission tomography study. Am. J. Psychiatr. 156, 252-257.

Kahneman, D. (2011). Thinking, Fast and Slow. Toronto: Doubleday Canada.

Latinjak, A. T., Hatzigeorgiadis, A., and Zourbanos, N. (2016). Goal-directed and spontaneous self-talk in anger- and anxiety-eliciting sport-situations. J. Appl. Sport Psychol. 29, 150-166. doi: 10.1080/10413200.2016.121 3330

Latinjak, A. T., Zourbanos, N., Lopez-Ros, V., and Hatzigeorgiadis, A. (2014). Goal-directed and undirected self-talk: exploring a new perspective for the study of athletes' self-talk. Psychol. Sport Exerc. 15, 548-558. doi: 10.1016/j.psychsport.2014.05.007

Leschziner, V. (2015). At the Chef's Table: Culinary Creativity in Elite Restaurants. Stanford, CA: Stanford University Press.

Leschziner, V., and Green, A. I. (2013). Thinking about food and sex: deliberate cognition in the routine practices of a field. Sociol. Theory 31, 116-144. doi: $10.1177 / 0735275113489806$ 
Lewin, K. (1936). Principles of Topological Psychology. Transl. by F. Heider and Grace M. Heider. New York, NY: McGraw Hill.

Lewin, K. (1939). Field theory and experiment in social psychology. Am. J. Sociol. 44, 868-896. doi: 10.1086/218177

Lizardo, O., Mowry, R., Sepulvado, B., Stoltz, D. S., Taylor, M. A., Van Ness, J., et al. (2016). What are dual process models? Implications for cultural analysis in sociology. Sociol. Theory 34, 287-310. doi: 10.1177/07352751166 75900

Lizardo, O., and Strand, M. (2010). Skills, toolkits, contexts and institutions: clarifying the relationship between different approaches to cognition in cultural sociology. Poetics 38, 205-228. doi: 10.1016/j.poetic.2009. 11.003

Markus, H. R., and Wurf, E. (1987). The dynamic self-concept: a social psychological perspective. Annu. Rev. Psychol. 38, 299-337. doi: 10.1146/annurev.ps.38.020187.001503

Martin, J. L. (2003). What is field theory? Am. J. Sociol. 109, 1-49. doi: $10.1086 / 375201$

Mead, G. H. (1934). Mind, Self, \& Society: From the Standpoint of a Social Behaviourist. ed Charles Morris. Chicago, IL: University of Chicago Press.

Mills, C. W. (1940). Situated actions and vocabulary of motive. Am. Sociol. Rev. 5, 904-913. doi: 10.2307/2084524

Moore, R. (2017). Fast or slow: sociological implications of measuring dual-process cognition. Sociol. Sci. 4, 196-223. doi: 10.15195/v4.a9

Parlett, M. (1991). Reflections on field theory. Br. Gestalt J. 1, 68-91.

Patterson, O. (2014). Making sense of culture. Annu. Rev. Sociol. 40, 1-30. doi: 10.1146/annurev-soc-071913-043123

Piiroinen, T. (2014). For 'central conflation': a critique of archerian dualism. Sociol. Theory 32, 79-99. doi: 10.1177/0735275114537632

Porpora, D. V. (2016). The recent methods debate in american sociology and how critical realism fits into it. J. Crit. Realism 15, 342-351. doi: 10.1080/14767430.2016.1193674

Pugh, A. (2013). What good are interviews for thinking about culture? Demystifying interpretive analysis. Am. J. Cult. Sociol. 1, 42-68. doi: 10.1057/ajcs.2012.4

Schonwetter, R. S., Walker, R. M., Kramer, D. R., and Robinson, B. E. (1993). Resuscitation decision making in the elderly: the value of outcome data. J. Gen. Intern. Med. 8, 295-300. doi: 10.1007/BF02 600139

Searle, J. R. (1995). The Social Construction of Reality. London: Penguin Books.

Seymour, J. (2003). Technology and 'natural death': a study of older people. Zeitschrift Gerontol. Geriatr. 36, 339-346.

Silver, D. (2011). The moodiness of action. Sociol. Theory 29, 199-222. doi: $10.1111 /$ j.1467-9558.2011.01394.x
Singer, P. A., Martin, D. K., and Kelner, M. (1999). Quality end-of-life care: patients' perspectives. J. Am. Med. Assoc. 281, 163-168.

Strauss, C. (2006). The imaginary. Anthropol. Theory 6, 322-344. doi: $10.1177 / 1463499606066891$

Swidler, A. (1986). Culture in action: symbols and strategies. Am. Sociol. Rev. 51, 273-286. doi: 10.2307/2095521

Swidler, A. (2001). Talk of Love: How Culture Matters. Chicago, IL: University of Chicago Press.

Vaisey, S. (2009). Motivation and justification: a dual-process model of culture in action. Am. J. Sociol. 114, 1675-1715. doi: 10.1086/597179

Vaisey, S. (2014). Is interviewing compatible with the dual-process model of culture? Am. J. Cult. Sociol. 2, 150-158. doi: 10.1057/ajcs.2013.8

Van Raalte, J. L., Vincent, A., and Brewer, B. W. (2016). Self-talk: review and sport-specific model. Psychol. Sport Exerc. 22: 139-148. doi: 10.1016/j.psychsport.2015.08.004

Vandrevala, T., Hampson, S. E., Daly, T., Arber, S., and Thomas, H. (2006). Dilemmas in decision-making about resuscitation-A focus group study of older people. Soc. Sci. Med. 62, 1579-1593. doi: 10.1016/j.socscimed.2005.08.038

Vaughan, D. (1996). The Challenger Launch Decision: Risky Technology, Culture and Deviance at NASA. Chicago, IL: University of Chicago Press.

Wheaton, B., and Gotlib, I. H. (1997). "Trajectories and turning points over the life course: concepts and themes," in Stress and Adversity Over the Life Course: Trajectories and Turning Points, eds B. Wheaton and I. H. Gotlib (New York, NY: Cambridge University Press), 1-26.

Wiley, N. (2016). Inner Speech and the Dialogical Self. Philadelphia, PA: Temple University Press.

Williams, L. H. (2017). Talk of pedophilia: the reflexive management of desire. Deviant Behav. 38, 1406-1418. doi: 10.1080/01639625.2016.1257880

Williams, L. H. (2018). Thinking about careers: reflexivity as bounded by previous, ongoing, and imagined experience. J. Crit. Realism 17, 46-62. doi: $10.1080 / 14767430.2018 .1438868$

Conflict of Interest Statement: The author declares that the research was conducted in the absence of any commercial or financial relationships that could be construed as a potential conflict of interest.

Copyright (C) 2018 Williams. This is an open-access article distributed under the terms of the Creative Commons Attribution License (CC BY). The use, distribution or reproduction in other forums is permitted, provided the original author(s) and the copyright owner(s) are credited and that the original publication in this journal is cited, in accordance with accepted academic practice. No use, distribution or reproduction is permitted which does not comply with these terms. 